\title{
Hepatocellular Carcinoma in Child-Pugh C Cirrhosis: Prognostic Factors and Survival Benefit of Nontransplant Treatments
}

\author{
Masatoshi Kudo ${ }^{a}$ Yukio Osaki ${ }^{b}$ Takashi Matsunagac Hiroshi Kasugaid

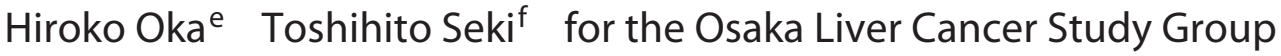 \\ ${ }^{a}$ Department of Gastroenterology and Hepatology, Kinki University School of Medicine, Osakasayama, \\ ${ }^{b}$ Department of Gastroenterology and Hepatology, Osaka Red Cross Hospital, Departments of ${ }^{\mathrm{C}}$ Medical \\ Informatics and ${ }^{\mathrm{d}}$ Gastrointestinal Oncology, Osaka Medical Center for Cancer and Cardiovascular Diseases, \\ eDepartment of Gastroenterology, Osaka City General Hospital, and f Department of Gastroenterology and \\ Hepatology, Kansai Medical University Takii Hospital, Osaka, Japan
}

\section{Key Words}

Hepatocellular carcinoma - Child-Pugh C cirrhosis .

Nontransplant treatment

\begin{abstract}
A retrospective multicenter study was conducted to clarify the survival benefit of nontransplant treatments for patients with hepatocellular carcinoma (HCC) associated with ChildPugh C cirrhosis. Data on 436 patients, including 203 treated patients with HCC, were collected from 20 institutions in Japan. Cox's proportional hazards model corrected for bias by propensity score analysis clearly showed the following as significant independent prognostic factors, including all four nontransplant treatments examined: transarterial chemoembolization, hepatic arterial infusion chemotherapy, percutaneous ethanol injection therapy, radiofrequency ablation, hepatitis B virus, number of tumors, log a-fetoprotein, encephalopathy, ascites and prothrombin time. The cumulative survival rate was significantly higher in the treated group than in the untreated group. The present findings suggest that prognosis can be improved by nontransplant treatments in patients with low Child-Pugh scores. Since this study was retrospective, the possibility of selection bias cannot be ruled out. Therefore, verification by a prospective controlled study is warranted.

(c) 2013 S. Karger AG, Basel
\end{abstract}

\section{Introduction}

The strong association of underlying chronic liver disease in patients with hepatocellular carcinoma (HCC) means that prognosis is largely determined not only by tumor stage, but also by liver function. Although both diseases are closely related, each is a major independent prognostic factor $[1,2]$. In patients with Child-Pugh C liver function, prognosis is extremely poor [3]. Therefore, most of the HCC practice guidelines, including the American Association for the Study of Liver Diseases, AASLD [4], the European Association for the Study of the Liver and the European Organisation for Research and Treatment of Cancer, EASL-EORTC [5], the Asian Pacific Association for the Study of the Liver [6], and the Japan Society of Hepatology, JSH (evidence based) [7], recommend liver transplantation for patients meeting the Milan criteria [8] and best supportive care for patients exceeding the criteria. Only the consensus-based treatment algorithm proposed by the JSH recommends transarterial chemoembolization (TACE) or ablation for HCC patients with Child-Pugh C liver function [9]. This retrospective multicenter study was conducted to clarify

Masatoshi Kudo and Yukio Osaki contributed equally to this study.

\section{KARGER}

E-Mail karger@karger.com

www.karger.com/ddi
(C) 2013 S. Karger AG, Basel

0257-2753/13/0316-0490\$38.00/0
Masatoshi Kudo, MD

Department of Gastroenterology and Hepatology

Kinki University School of Medicine, 337-2 Ohno-Higashi

Osakasayama, Osaka 589-8511 (Japan)

E-Mail m-kudo@med.kindai.ac.jp 
Fig. 1. Cumulative survival rate by treat-

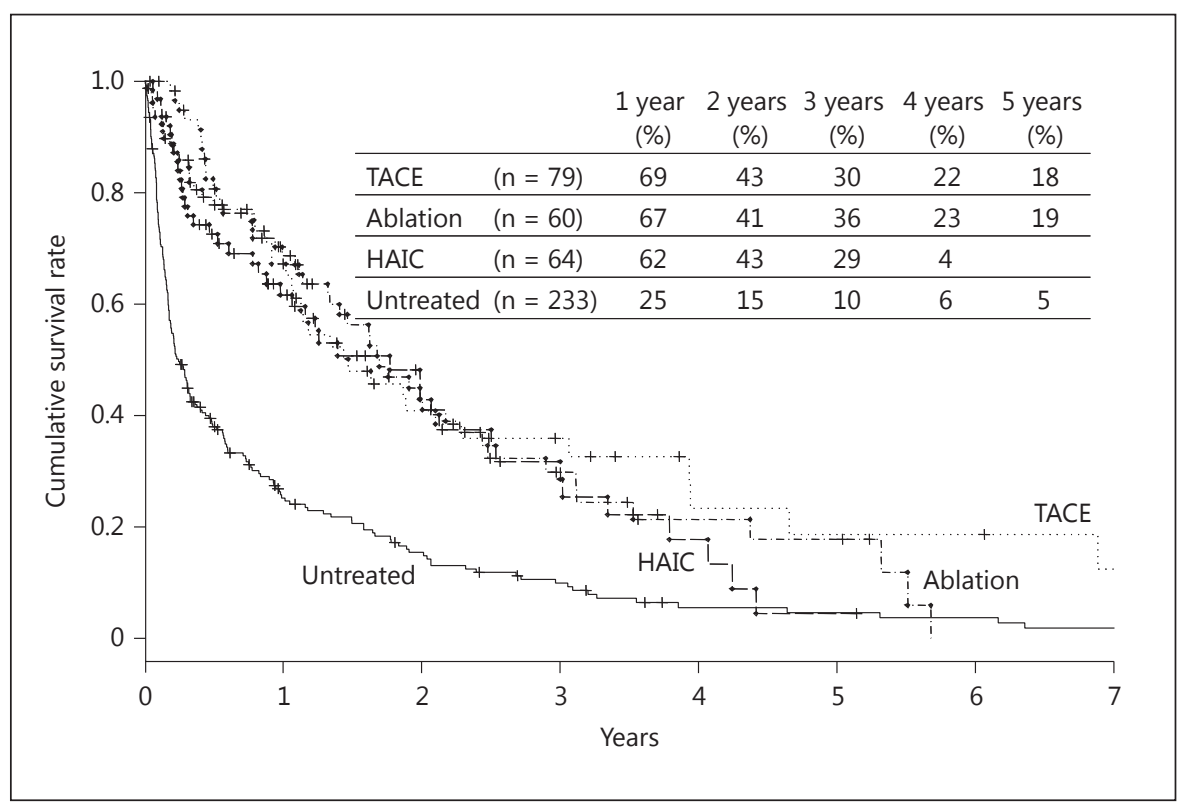
ment.

the prognostic factors and survival benefit of nontransplant treatment for HCC patients with Child-Pugh C cirrhosis.

\section{Patients and Methods}

Patients with underlying Child-Pugh C cirrhosis who were histologically or clinically diagnosed with HCC by diagnostic imaging and tumor markers during the 10-year period from 1994 to 2003 were assessed. A total of 454 patients were collected from 20 institutions in Japan. After excluding 18 patients who underwent liver transplantation or whose date of death or last day of follow-up was missing, 436 patients were left for analysis.

To clarify the prognostic factors, multivariate analysis was performed using Cox's proportional hazards model corrected for bias by propensity score weighting. The cumulative survival rate was calculated based on the time of diagnosis to the time of death and compared between patients who received nontransplant treatments (treated group) and those who did not (untreated group) according to Child-Pugh score, tumor size, number of tumors, tumor stage, and meeting or exceeding the Milan criteria. Observation was started at the time of HCC diagnosis and was continued until March 31, 2005. Cumulative survival rates were compared by Kaplan-Meier analysis and the log-rank test. Statistical significance was set at $\mathrm{p}<0.05$.

\section{Results}

\section{Prognostic Factors}

Multivariate analysis was performed for 17 of the 23 factors investigated, excluding those irrelevant to prog-
Table 1. Predictive factors of HCC associated with Child-Pugh C liver cirrhosis

\begin{tabular}{|c|c|c|c|c|c|}
\hline & Coef. & $\operatorname{Exp}$ (coef.) & SE(coef.) & $\mathrm{z}$ & $\mathrm{p}$ \\
\hline TACE & -0.539 & 0.583 & 0.199 & -2.71 & 0.007 \\
\hline HAIC & -1.049 & 0.350 & 0.235 & -4.47 & $<0.001$ \\
\hline PEIT & -0.536 & 0.585 & 0.219 & -2.45 & 0.014 \\
\hline RFA & -0.626 & 0.535 & 0.293 & -2.14 & 0.032 \\
\hline $\mathrm{HBV}$ & 0.392 & 1.481 & 0.178 & 2.20 & 0.028 \\
\hline Tumors (n) & 0.073 & 1.076 & 0.034 & 2.17 & 0.030 \\
\hline $\log$ AFP & 0.247 & 1.280 & 0.059 & 4.17 & $<0.001$ \\
\hline Encephalopathy & -0.507 & 0.603 & 0.101 & -5.02 & $<0.001$ \\
\hline Ascites & -0.469 & 0.626 & 0.118 & -3.98 & $<0.001$ \\
\hline PT & 0.018 & 1.018 & 0.005 & 3.49 & $<0.001$ \\
\hline Fit $^{1}$ & -2.449 & 0.086 & 0.391 & -6.27 & $<0.001$ \\
\hline
\end{tabular}

Results of Cox's proportional hazards model corrected for bias by propensity score weighting. $\mathrm{HBV}=$ Hepatitis $\mathrm{B}$ virus; $\mathrm{AFP}=$ a-fetoprotein; PT $=$ prothrombin time.

${ }^{1}$ Propensity score.

nosis, and the following significant independent factors were extracted: all medical treatments including TACE, hepatic arterial infusion chemotherapy (HAIC), percutaneous ethanol infection therapy (PEIT), percutaneous radiofrequency ablation (RFA), negative hepatitis $B$ virus, small number of tumors, low $\log \alpha$-fetoprotein values, no encephalopathy, no ascites and high prothrombin time values (table 1). 
Fig. 2. Cumulative survival rate by ChildPugh score of 10 or 11 points.

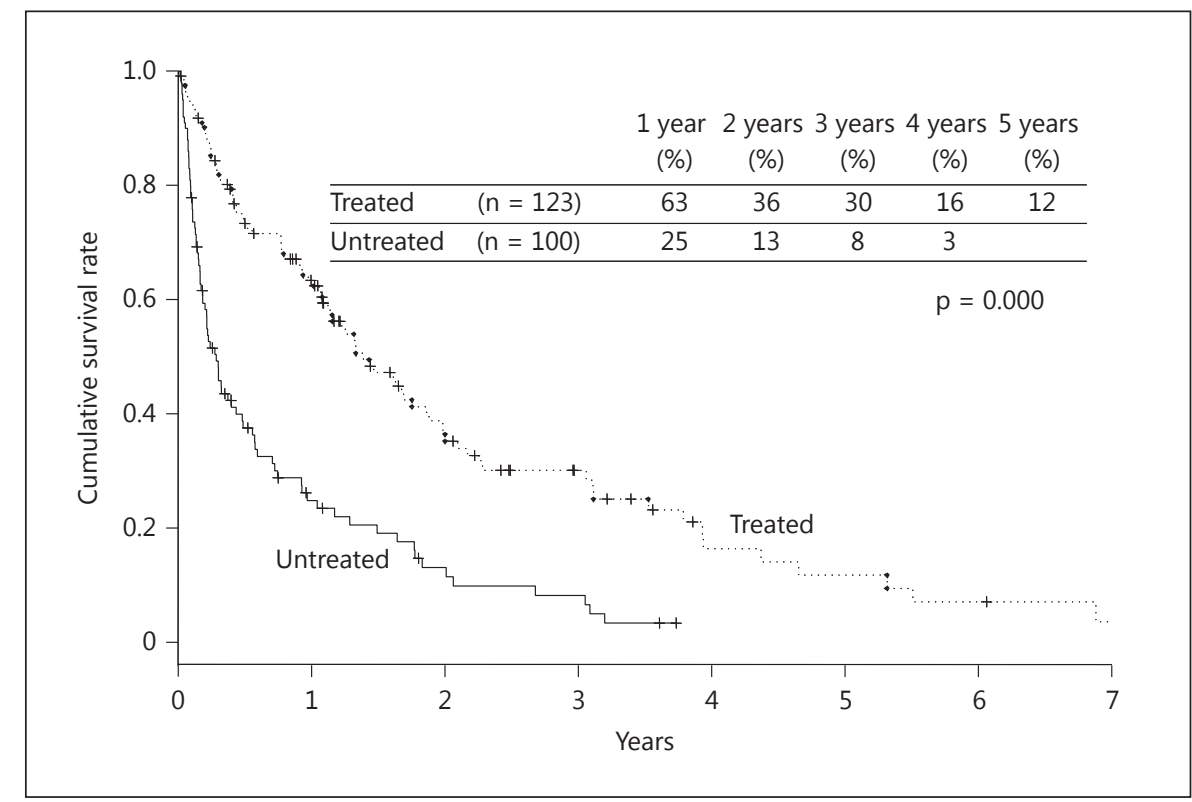

Fig. 3. Cumulative survival rate by ChildPugh score of 12 or 13 points.

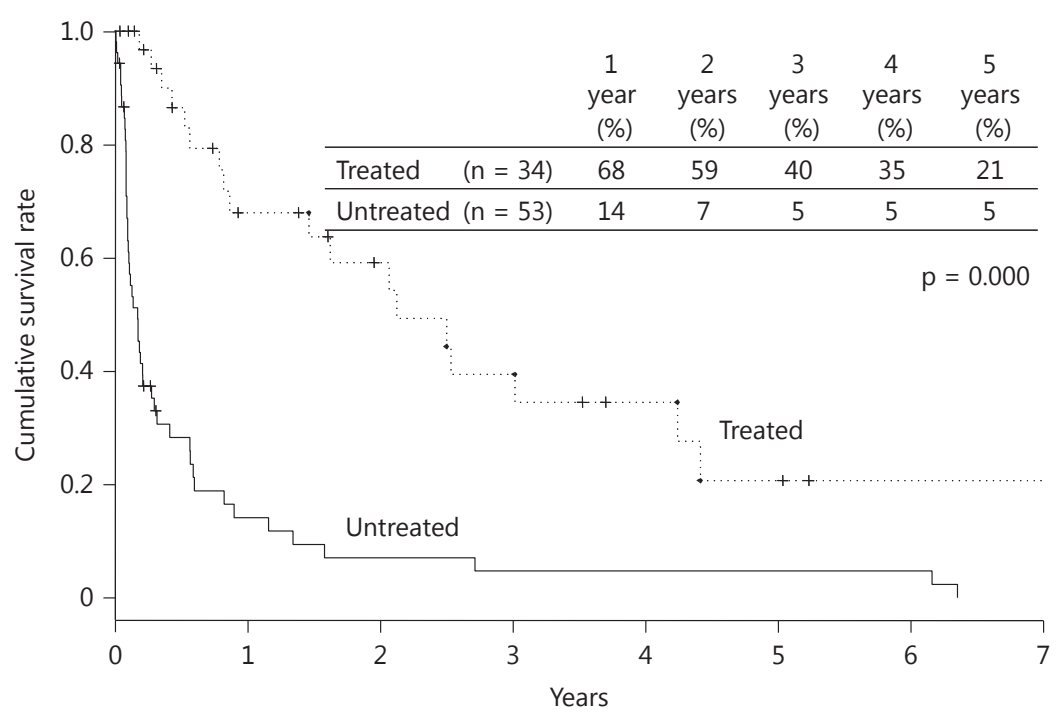

\section{Cumulative Survival Rate Classified by Treatment} Modalities

The cumulative survival rate in treated patients was $69 \%$ ( 1 year), $30 \%$ ( 3 years) and $18 \%$ ( 5 years) in the TACE group ( $\mathrm{n}=79), 62,29$ and $4 \%$, respectively, in the HAIC group $(n=64)$, and 67,36 and $19 \%$, respectively, in the local ablation group. The survival rate was significantly higher in all three of these treated groups than in the un- treated group $[\mathrm{n}=233 ; 25 \%$ ( 1 year), 10\% (3 years) and $5 \%$ (5 years); fig. 1$]$.

\section{Prognosis Classified by Child-Pugh Score}

Patients were divided into the following 3 groups based on their Child-Pugh score: 10-11 points, 12-13 points and 14-15 points. The cumulative survival rate in all three of these treated groups was significantly 
Fig. 4. Cumulative survival rate by ChildPugh score of 14 or 15 points.

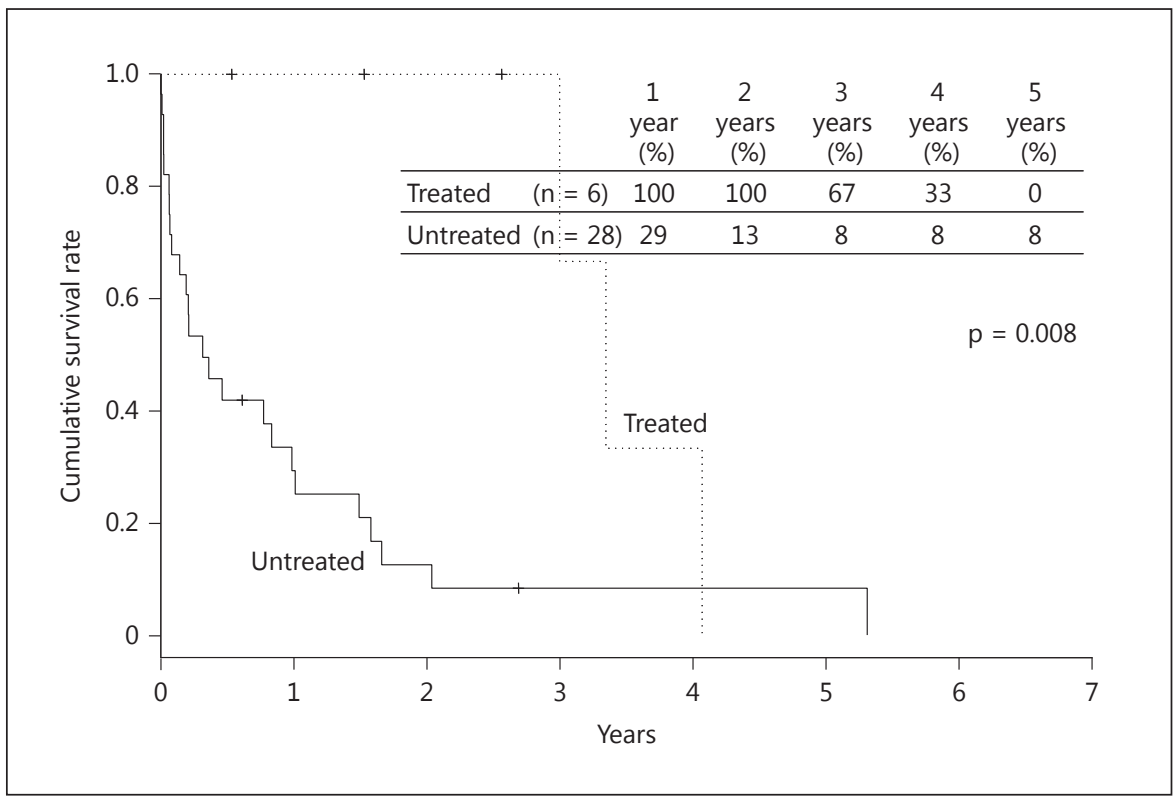

Fig. 5. Cumulative survival rate by tumor size $\leq 2 \mathrm{~cm}$.

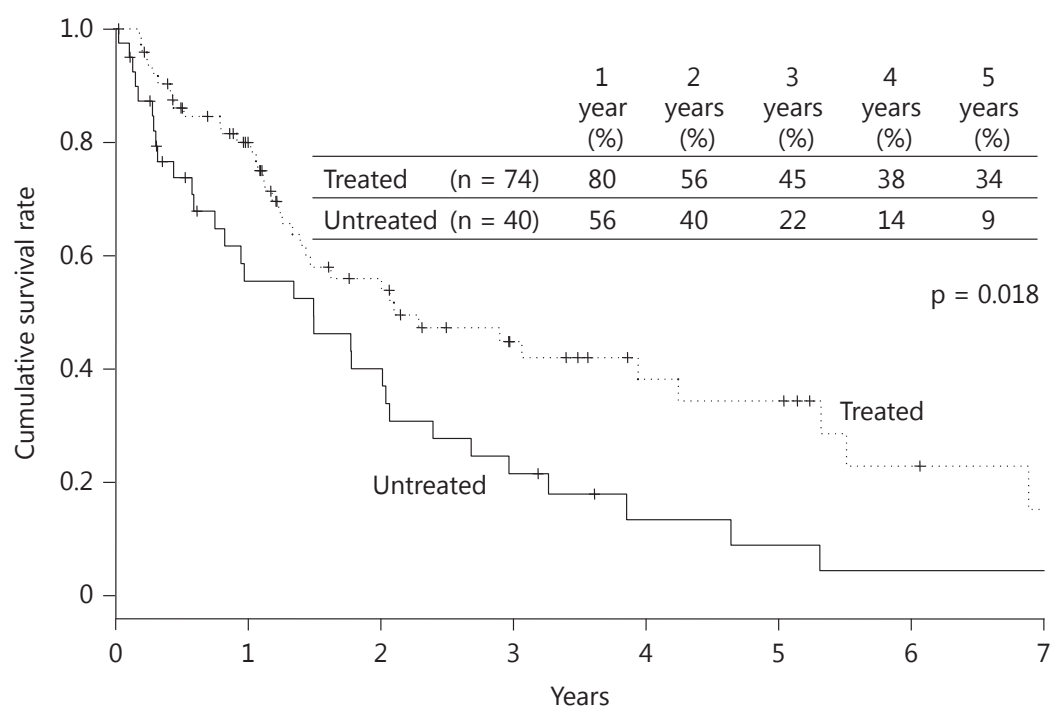

higher than in the untreated group (fig. 2-4). However, only 6 patients in the 14 - to 15 -point group received treatment.

\section{Prognosis Classified by Tumor Size and Number of \\ Tumors}

The cumulative survival rate was significantly higher in the treated group than in the untreated group for pa- tients with tumors $\leq 2 \mathrm{~cm}$ in diameter (fig. 5), those with tumors $>2$ to $\leq 5 \mathrm{~cm}$ in diameter (fig. 6), and those with tumors $>5 \mathrm{~cm}$ in diameter (fig. 7).

Similarly, the cumulative survival rate in the treated group was significantly higher in patients with a solitary tumor [ $76 \%$ (1 year), $40 \%$ ( 3 years) and $26 \%$ ( 5 years)] than in the untreated group $(37,11$ and $0 \%$, respectively), in patients with 2 or 3 tumors $(68,29$ and $12 \%$, respectively) than 
Fig. 6. Cumulative survival rate by tumor size $>2$ and $\leq 5 \mathrm{~cm}$.

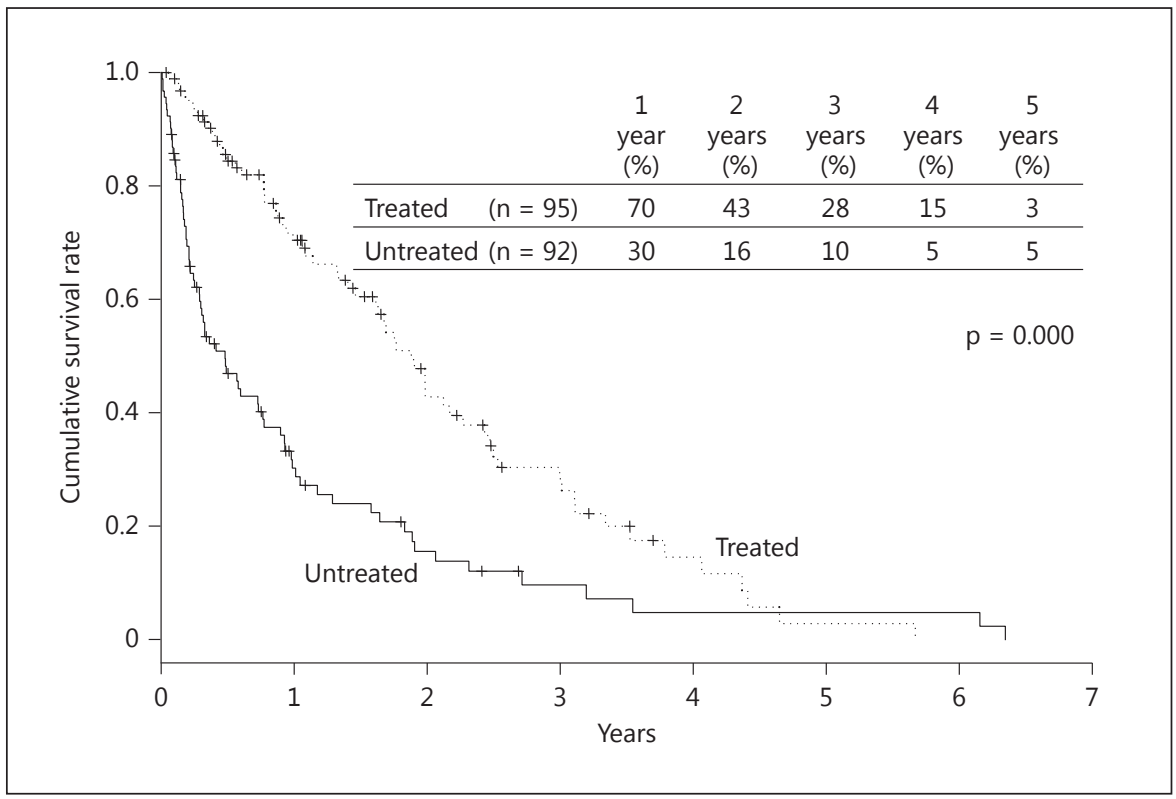

Fig. 7. Cumulative survival rate by tumor size $>5 \mathrm{~cm}$.

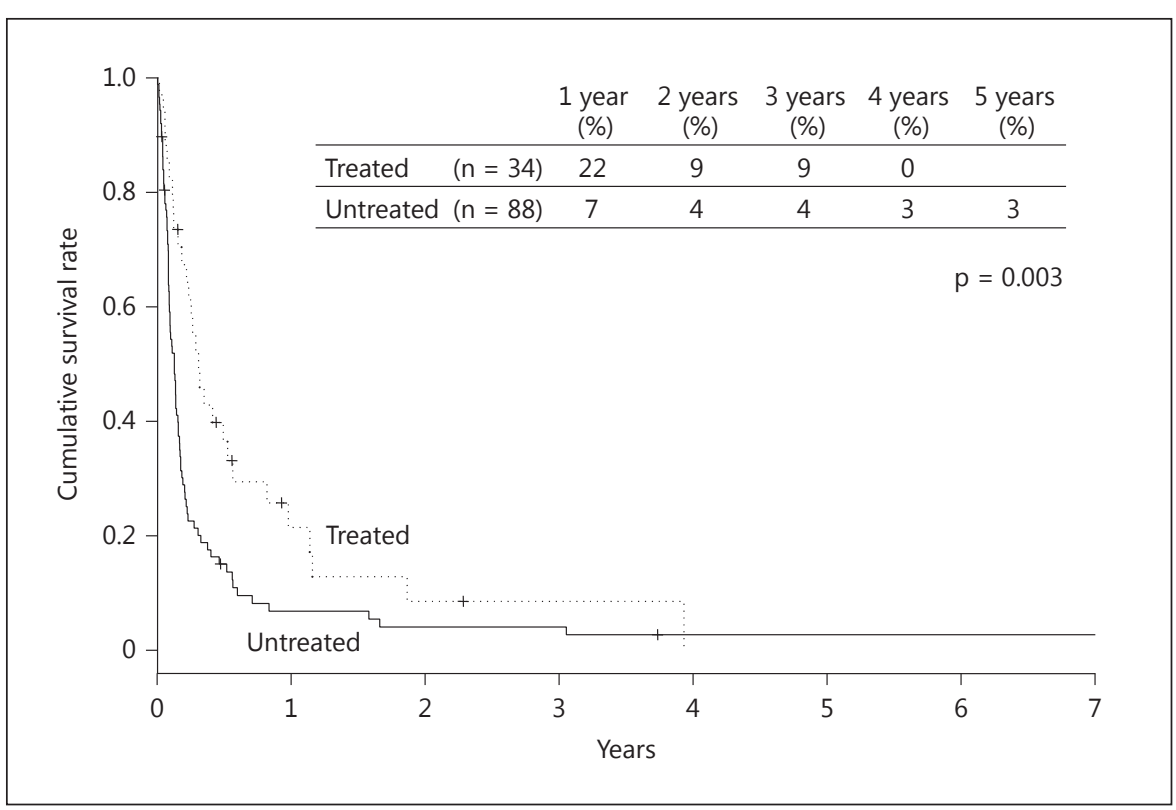

in the untreated group $(29,11$ and $7 \%$, respectively), and in patients with $>3$ tumors $(48,22$ and $4 \%$, respectively) than in the untreated group $(15,8$ and $6 \%$, respectively).

\section{Prognosis Classified by TNM Stage by the Liver}

Cancer Study Group of Japan

All patients were classified into stage I-IV according to their TNM stage by the Liver Cancer Study Group of
Japan $[1,2]$. The cumulative survival rate was significantly higher in the treated group than in the untreated group for stage I patients [ $85 \%$ ( 1 year), $51 \%$ ( 3 years) and 38\% (5 years); fig. 8], stage II patients (fig. 9), and stage III or stage IV patients (fig. 10, 11). 
Fig. 8. Cumulative survival rate by TNM stage I.

Fig. 9. Cumulative survival rate by TNM stage II.
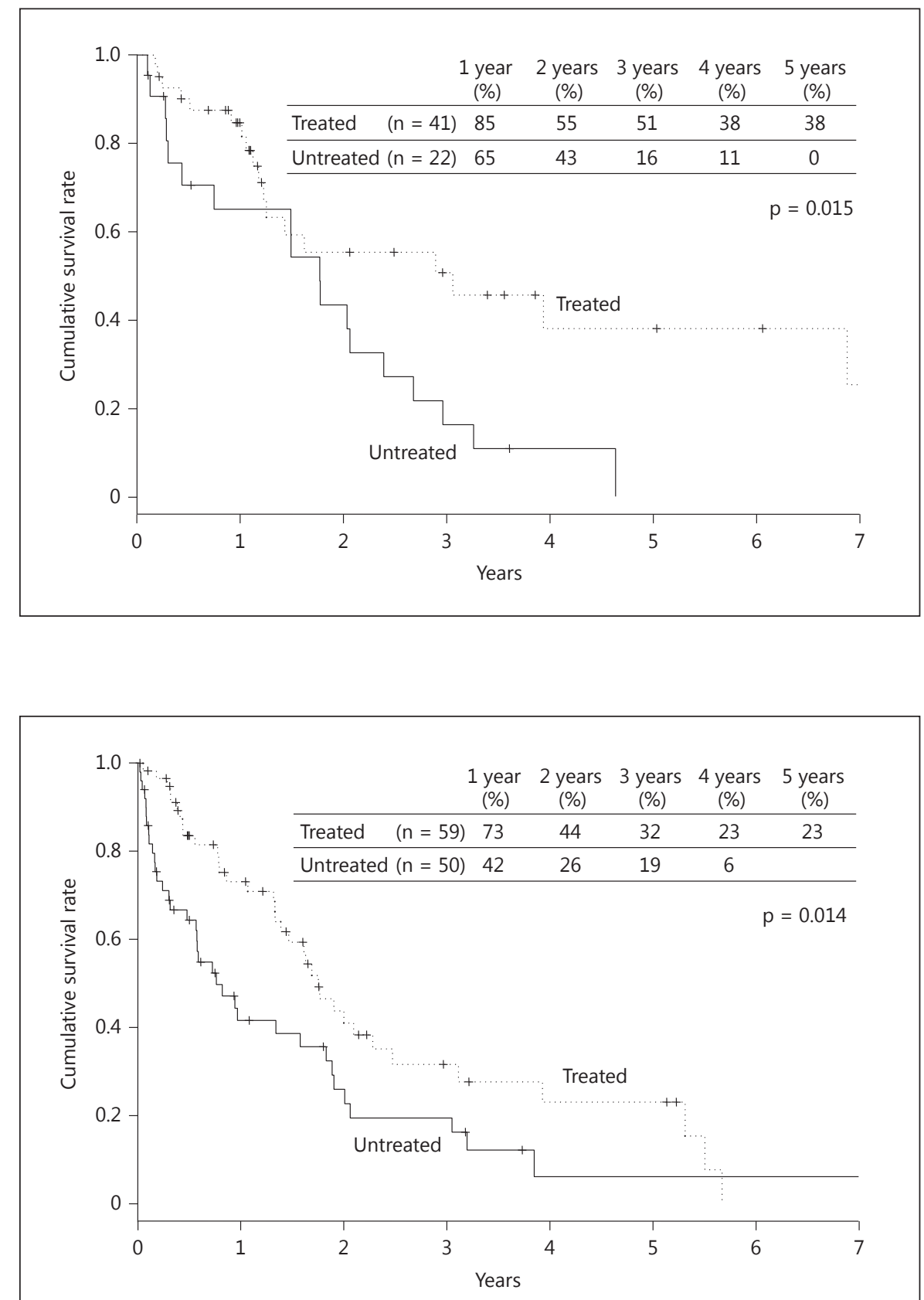

Prognosis Classified by $\leq 3$ Tumors $\leq 3 \mathrm{~cm}$ in Diameter and Tumors Meeting or Exceeding the Milan Criteria The cumulative survival rate in the treated group was significantly higher in patients with $\leq 3$ tumors $\leq 3 \mathrm{~cm}$ in diameter [ $79 \%$ ( 1 year), 40\% (3 years) and 30\% (5 years)] than in the untreated group (54, 17 and $6 \%$, respectively). Similarly, the cumulative survival rate in the treated group was significantly higher in patients with $\geq 3$ tumors or with tumors $\geq 3 \mathrm{~cm}$ in diameter than in the untreated group.

The cumulative survival rate was significantly higher in the treated group than in the untreated group for patients meeting the Milan criteria [8] ( $\leq 3$ tumors $\leq 3 \mathrm{~cm}$ in diameter or a solitary tumor $\leq 5 \mathrm{~cm}$ in diameter; fig. 12) and was also higher for patients exceeding the criteria (fig. 13). 
Fig. 10. Cumulative survival rate by TNM stage III.

Fig. 11. Cumulative survival rate by TNM stage IV.
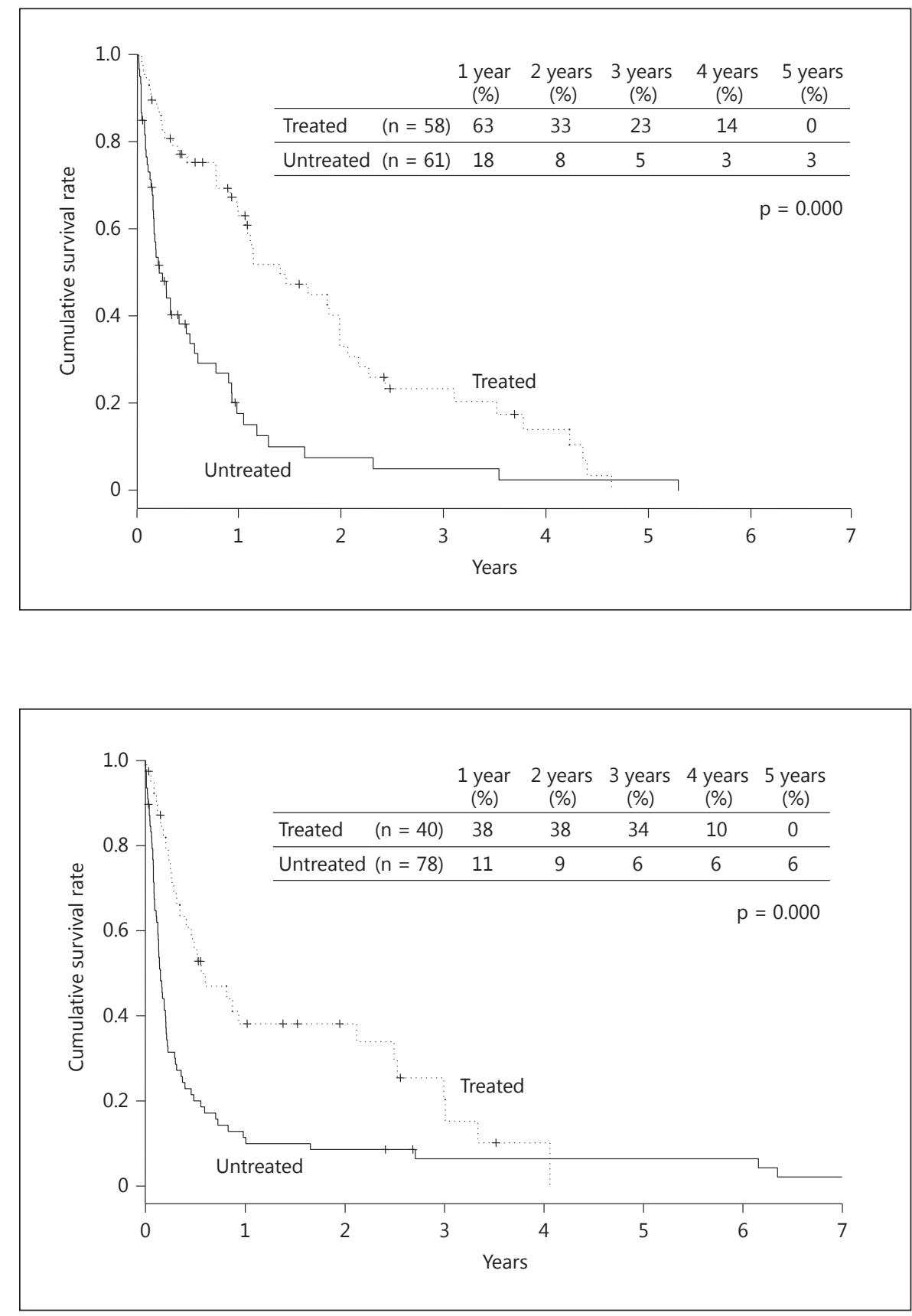

\section{Discussion}

The most important goal of cancer treatment is to improve patient prognosis. The prognosis of HCC is often determined by the stage of liver cirrhosis or the deterioration of liver function associated with cancer treatment, irrespective of tumor presence or stage. Therefore, to determine the treatment indications and select appropriate treatment, comprehensive judgments should be made considering both factors carefully.

Patients with Child-Pugh $\mathrm{C}$ disease are generally not treated, regardless of tumor stage, because of their poor prognosis. As mentioned earlier, according to the treatment algorithm recommended by the AASLD and EASLEORTC, patients with Child-Pugh C liver function should be regarded as having end-stage (terminal) disease and giv- 
Fig. 12. Cumulative survival rate by meeting the Milan criteria.

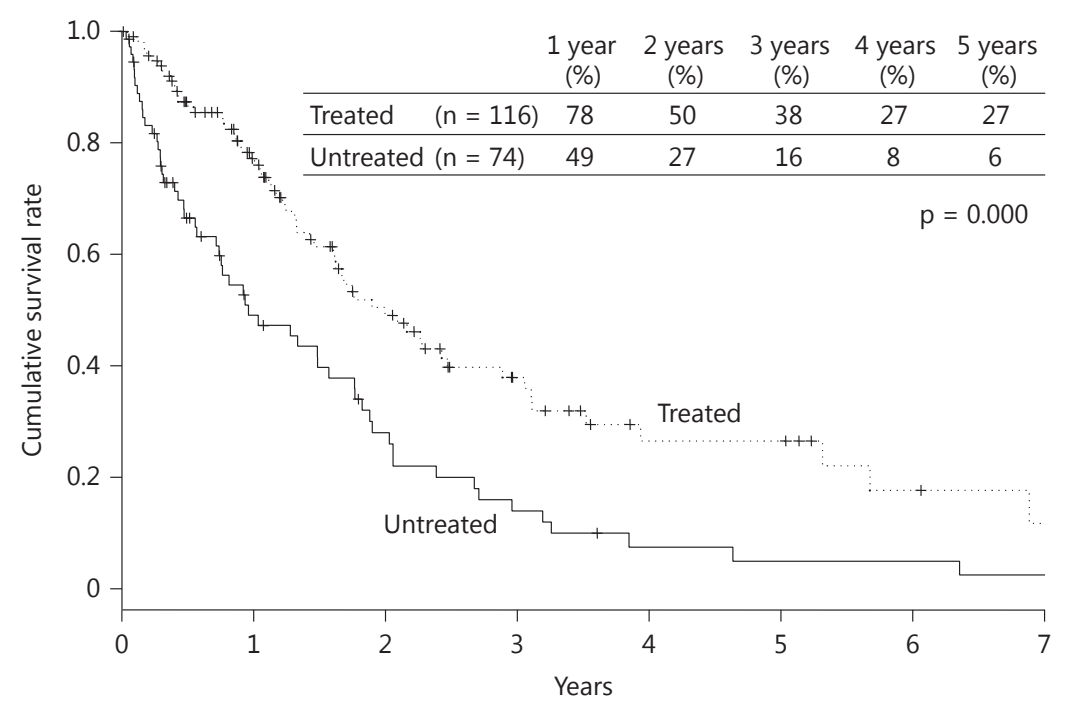

Fig. 13. Cumulative survival rate by exceeding the Milan criteria.

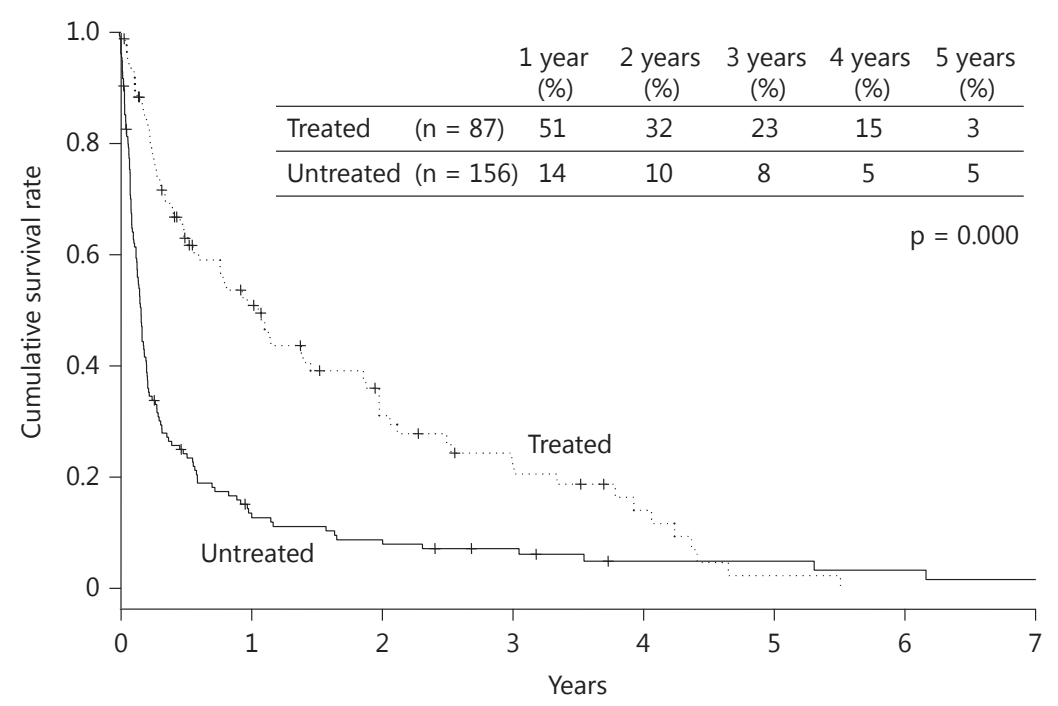

en only symptomatic treatment. With respect to HCC associated with Child-Pugh $\mathrm{C}$ disease, the evidence-based guidelines for the treatment of liver cancer published by the JSH [7] recommend liver transplantation [10] for patients meeting the Milan criteria, and palliative therapy for all other patients. However, even in patients with HCC meeting the Milan criteria, liver transplantation is not a standard in Japan because of the shortage of brain-death donors.
This study revealed several interesting findings. One such finding is that only around half (53\%) of the patients included in the analysis received some kind of medical treatment. The most notable findings, however, are that all nontransplant treatments (TACE [11], PEIT [12, 13], RFA and HAIC [14]) were independent prognostic factors and that survival was better in patients who received medical treatment than in those who did not. In view of 
the current state of clinical practice, it can be presumed that some kind of treatment is being administered to patients with HCC associated with Child-Pugh $\mathrm{C}$ cirrhosis and that the treatment might be contributing to an improved prognosis in a limited number of treated patients. An unexpected finding was that so many patients received treatment, with significantly better outcome in treated patients than in untreated patients. However, since the present study is retrospective, it is possible that only patients with relatively good liver function were treated as they were expected to respond to treatment; as a result, those who were not considered able to tolerate treatment were likely placed in the untreated group. Therefore, a strong selection bias may have been present in this study. Nevertheless, it is clearly evident that a considerable number of patients with HCC associated with Child-Pugh C cirrhosis received and responded to cancer treatment. Therefore, treatment may improve prognosis.

Assessment of prognosis in the three groups with different Child-Pugh scores revealed that survival was better in the treated than in the untreated patients. However, the 14- to 15-point group had only a small number of treated patients $(n=6)$, so this finding does not necessarily indicate the superiority of the treatment in this group. Patients in the treated group who met the Milan criteria or the general criteria for local ablation ( $\leq 3$ tumors $\leq 3 \mathrm{~cm}$ in diameter) showed a higher survival rate throughout the study period. Interestingly, patients with HCCs exceeding the Milan criteria in the treatment group also showed a better survival than untreated patients. This may be attributed to the frequent use of superselective TACE even in multinodular HCC patients in Japan, which does not cause deterioration of liver function. A previous report also described that superselective TACE was identified as having survival benefit in HCC patients with Child-Pugh C [15].

These results suggest that prognosis may be improved by treating eligible patients with early-stage tumors for radical cure. Despite the poor prognosis of Child-Pugh C disease, the present results show that treatment might improve prognosis in patients with low Child-Pugh scores, which is in agreement with the results reported by Nouso et al. [15].

In conclusion, this retrospective multicenter study of 436 HCC patients with Child-Pugh C cirrhosis showed that nontransplant medical treatments (i.e. TACE, RFA, PEIT and HAIC) confer survival benefit. In addition, medical treatment should be considered for HCC patients, especially for patients with low Child-Pugh scores. Finally, this result confirmed the validity of recommendation by the consensus-based JSH treatment algorithm [9].

\section{Disclosure Statement}

The authors have no conflicts of interest to declare.

\section{References}

1 Kudo M, Chung H, Osaki Y: Prognostic staging system for hepatocellular carcinoma (CLIP score): its value and limitations, and a proposal for a new staging system, the Japan Integrated Staging Score (JIS score). J Gastroenterol 2003;38:207-215.

-2 Kudo M, Chung H, Haji S, Osaki Y, Oka H, Seki T, Kasugai H, Sasaki Y, Matsunaga T: Validation of a new prognostic staging system for hepatocellular carcinoma: the JIS score compared with the CLIP score. Hepatology 2004;40:1396-1405.

-3 Pugh RN, Murray-Lyon IM, Dawson JL, Pietroni MC, Williams R: Transection of the oesophagus for bleeding oesophageal varices. $\mathrm{Br}$ J Surg 1973;60:646-649.

4 Bruix J, Sherman M: Management of hepatocellular carcinoma: an update. Hepatology 2011;53:1020-1022.

5 Llovet JM, Ducreux M: EASL-EORTC clinical practice guidelines: management of hepatocellular carcinoma. J Hepatol 2012;56:908943.
6 Omata M, Lesmana LA, Tateishi R, Chen PJ, Lin SM, Yoshida H, Kudo M, Lee JM, Choi BI, Poon RT, et al: Asian Pacific Association for the Study of the Liver consensus recommendations on hepatocellular carcinoma. Hepatol Int 2010;4:439-474.

7 Makuuchi M, Kokudo N, Arii S, Futagawa S, Kaneko S, Kawasaki S, Matsuyama Y, Okazaki M, Okita K, Omata M, et al: Development of evidence-based clinical guidelines for the diagnosis and treatment of hepatocellular carcinoma in Japan. Hepatol Res 2008;38:37-51.

8 Mazzaferro V, Regalia E, Doci R, Andreola S, Pulvirenti A, Bozzetti F, Montalto F, Ammatuna M, Morabito A, Gennari L: Liver transplantation for the treatment of small hepatocellular carcinomas in patients with cirrhosis. N Engl J Med 1996;334:693-699.

$>9$ Kudo M, Izumi N, Kokudo N, Matsui O, Sakamoto M, Nakashima O, Kojiro M, Makuuchi M: Management of hepatocellular carcinoma in Japan: consensus-based clinical practice guidelines proposed by the Japan So- ciety of Hepatology (JSH) 2010 updated version. Dig Dis 2011;29:339-364.

10 Belghiti J, Fuks D: Liver resection and transplantation in hepatocellular carcinoma. Liver Cancer 2012;1:71-82.

-11 Lencioni R: Chemoembolization in patients with hepatocellular carcinoma. Liver Cancer 2012;1:41-50.

-12 Lin S, Hoffmann K, Schemmer P: Treatment of hepatocellular carcinoma: a systematic review. Liver Cancer 2012;1:144-158.

13 Lin SM: Local ablation for hepatocellular carcinoma in Taiwan. Liver Cancer 2013;2:7383.

14 Kudo M: Treatment of advanced hepatocellular carcinoma with emphasis on hepatic arterial infusion chemotherapy and molecular targeted therapy. Liver Cancer 2012;1:62-70.

15 Nouso K, Ito Y, Kuwaki K, Kobayashi Y, Nakamura S, Ohashi Y, Yamamoto K: Prognostic factors and treatment effects for hepatocellular carcinoma in Child C cirrhosis. Br J Cancer 2008;98:1161-1165. 